\title{
$\mathrm{N}$-杂环卡宾催化对氟硝基苯和苯甲醛的亲核酰基化反应研究
}

\author{
于凤丽 ${ }^{a}$ 鲁玉倩 ${ }^{a}$ 袁 冰 ${ }^{a}$ 解从霞 $*, a$ 于世涛 ${ }^{b}$ \\ ( ${ }^{a}$ 青岛科技大学 生态化工国家重点实验室培育基地 青岛 266042) \\ ( ${ }^{b}$ 青岛科技大学化工学院 青岛 266042)
}

\begin{abstract}
摘要 以咪唑类离子液体为 $\mathrm{N}$-杂环卡宾 $(\mathrm{NHC})$ 催化剂的前体, 应用于催化芳基氟化物和酲的亲核酰基化反应, 成功地 将酰基直接引入到缺电子的芳环上，制备出含吸电子基团的芳香酮. 以对氟硝基苯和苯甲醛的反应制备对硝基二苯甲 酮为模板, 详细探讨了 $\mathrm{NHC}$ 催化的亲核酰基化反应的机理和反应规律, 优化出最佳反应条件, 并考察了催化剂的循环 使用效果. 实验结果表明, 以 DMSO 为溶剂、离子液体 $1\left(\mathrm{R}=n-\mathrm{C}_{12} \mathrm{H}_{25}\right)$ 为催化剂前体、叔丁醇钾为碱, 离子液体的用 量为对氟硝基苯的 $33 \mathrm{~mol} \%$, 离子液体和叔丁醇钾的物质的量比为 $1: 4$, 反应温度为 $30{ }^{\circ} \mathrm{C}$, 反应时间为 $6 \mathrm{~h}$, 对氟硝基 苯的转化率可达 $93.0 \%$, 对硝基二苯甲酮的收率为 $88.6 \%$. 离子液体循环使用 4 次，对硝基二苯甲酮的收率仍在 75\%以 上.
\end{abstract}

关键词＼cjkstart亲核酰基化; $\mathrm{N}$-杂环卡宾; 极性反转; 对氟硝基苯; 对硝基二苯甲酮

\section{Nucleophilic Acylation of 4-Fluoronitrobenzene with Benzaldehyde Catalyzed by N-Heterocyclic Carbene}

\author{
Yu, Fengli ${ }^{a}$ \\ Lu, Yuqian ${ }^{a} \quad$ Yuan, Bing $^{a}$ \\ Xie, Congxia*,a \\ Yu, Shitao ${ }^{b}$ \\ ( ${ }^{a}$ State Key Laboratory Base of Eco-chemical Engineering, Qingdao University of Science and Technology, Qingdao 266042) \\ ( ${ }^{b}$ College of Chemical Engineering, Qingdao University of Science and Technology, Qingdao 266042)
}

\begin{abstract}
Imidazolium ionic liquids as the catalyst precursor of N-heterocyclic carbene (NHC) have been applied to catalyze the nucleophilic acylation of arylfluoride with aromatic aldehyde or aliphatic aldehyde. The acyl group can be directly introduced to the aromatic ring with an electron-withdrawing group. The reaction of 4-fluoronitrobenzene with benzaldehyde for the preparation of 4-nitrobenzophenone is selected as the model reaction. The mechanism of nucleophilic acylation catalyzed by NHC is proposed. The ionic liquid firstly is converted to NHC under the presence of alkali. NHC makes benzaldehyde carry through the umpolung, and the acyl of benzaldehyde as the nucleophilic group attacks the carbon atom bearing fluorine of 4-fluoronitrobenzene, followed by loss of the fluorine as an anion. Base-promoted eliminations of a proton and NHC from the intermediate take place to afford the target product of 4-nitrobenzophenone. The effects of the alkyl chain of the ionic liquid, the amount of ionic liquid, different solvent, the type of alkali, the amount of alkali, reaction temperature and reaction time on the catalytic reaction have been fully investigated. The selected optimal conditions are as follows: DMSO as the solvent, the ionic liquid $1\left(\mathrm{R}=n-\mathrm{C}_{12} \mathrm{H}_{25}\right)$ as the catalyst precursor, potassium tert-butoxide as the alkali, the amount of the ionic liquid 33 mol\%, the molar ratio of the ionic liquid to the alkali $1: 4$, reaction temperature $30{ }^{\circ} \mathrm{C}$ and reaction time $6 \mathrm{~h}$. Under the above optimal conditions, the conversion of 4 -fluoronitrobenzene is $93.0 \%$, and the yield of 4-nitrobenzophenone is $88.6 \%$. After the reaction, the ionic liquid is dried under vacuum and then can be directly reused. The recycling efficiency of the ionic liquid is investigated. The result showed that the yield of 4-nitrobenzophenone still could reach above $75 \%$ after 4 recycles of the ionic liquid. The study provides a new environmentally-friendly method for the direct introduction of the acyl group to the electron-deficient aromatic ring to synthesize the aromatic ketones with electron-withdrawing group.
\end{abstract}

Keywords nucleophilic acylation; N-heterocyclic carbene; umpolung; 4-fluoronitrobenzene; 4-nitrobenzophenone

*E-mail: xiecongxia@126.com

Received March 24, 2015; revised May 26, 2015; published online June 4, 2015.

Project supported by the National Natural Science Foundation of China (No. 21006055) and the Beijing National Laboratory for Molecular Sciences (No. 20140141).

国家自然科学基金(No. 21006055)和北京分子科学国家实验室开放课题(No. 20140141)资助项目. 
1832 年, Wöhler 和 Liebig ${ }^{[1]}$ 发现氧根负离子 $\left(\mathrm{CN}^{-}\right)$ 可以催化苯甲醛的安息香缩合反应. 直到 1903 年, Lapworth $^{[2]}$ 对其催化机理进行了研究, 提出了极性反转 的催化机理. 1943 年, Ukai 等 ${ }^{[3]}$ 发现辅酶维生素 B1 在碱 存在下生成 N-杂环卡宾(N-Heterocyclic Carbene, NHC), 与氰根负离子类似, 也可以催化安息香缩合反应, 并且 其操作简单、污染小, 极大地降低了对人体的毒害. 随 后, 化学家们开始尝试合成类似维生素 B1 结构的卡宾 催化剂. 1991 年, Arduengo 等 ${ }^{[4]}$ 首次分离出第一个稳定 的 N-杂环卡宾(咪唑-2-碳烯), 推动了 NHC 的飞速发展. $\mathrm{NHC}$ 具有很强的亲核性, 近年来, 其作为小分子催化剂 引起了科学家们的广泛关注 $\left.{ }^{[5} 10\right]$. 目前, NHC 已在安息 香缩合反应 ${ }^{[1 \sim 13]} 、$ Stetter 反应 ${ }^{[14 \sim 16]} 、 \alpha, \beta$-不饱醛酮的共 价极性反转反应 ${ }^{[17 \sim 20]}$ 、酯交换反应 ${ }^{[21 ~ 23]}$ 等中具有较广 泛的应用.

众所周知, 芳香酮是一类重要的精细化学品和中间 体, 其主要通过 Friedel-Crafts 酰基化反应合成. FriedelCrafts 酰基化反应是一类重要的芳香族亲电取代反应, 因此芳环上的电子云密度越大, 反应越容易进行. 若芳 环上含有 $-\mathrm{NO}_{2},-\mathrm{SO}_{3} \mathrm{H},-\mathrm{COOH},-\mathrm{COR}$ 等吸电子基团时, 使芳环上的电子云密度降低, 酰基化反应很难进行. 另 一方面, 目前 Friedel-Crafts 酰基化反应主要使用 $\mathrm{AlCl}_{3}$ 等 Lewis 酸或浓硫酸等 Brønsted 酸性催化剂, 这些催化 剂都存在一定的缺点, 如稳定性差、用量大、腐蚀严重、 环境污染大等, 特别是都存在难以分离回收和重复使用 的问题. 因此, 将酰基直接引入到芳环的缺电子位置, 制备含吸电子基团的芳香酮化合物, 通过正常的 Friedel-Crafts 酰基化反应是很难实现的, 需要寻找环境 友好的新途径.

2003 年, Suzuki 等 ${ }^{[24]}$ 报道了咪唑盐在碱存在下催化 芳基氟化物和芳香醛的反应, 成功地将酰基引入到含有 吸电子基团的芳香化合物中. 他们提出了 $\mathrm{NHC}$ 催化的 极性反转和非常规的亲核酰基化反应的机理, 在该机理 中, 芳香醛经过极性反转后作为亲核试剂进攻芳基氟化 物，因此芳基氟化物的芳环上含有吸电子基团时，对反 应是有利的. 随后, Suzuki 等 ${ }^{[25,26]}$ 把这一类型的反应应 用于氧杂葸酮和呋啶酮的合成. 可见, NHC 催化的亲核 酰基化反应是将酰基直接引入到缺电子芳环上的一个 非常有效的途径.

本文合成了五种咪唑类离子液体 $\mathbf{1}$, 将其作为 $\mathrm{NHC}$ 催化剂的前体, 应用于催化芳基氟化物和醛的亲核酰基 化反应. 以对氟硝基苯和苯甲酫的反应为模板, 成功地 制备出对硝基二苯甲酮, 并实现了催化剂的循环利用. 为直接将酰基引入到缺电子的芳环上、制备含有吸电子 基团的芳香酮提供了一条环境友好的新途径.

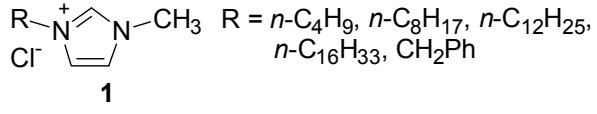

\section{1 结果与讨论}

\section{1 催化机理}

咪唑类离子液体 1 在碱的作用下, 催化对氟硝基苯 和苯甲醛发生亲核酰基化反应生成对硝基二苯甲酮，其 催化机理如图 1 所示. 离子液体 $\mathbf{1}$ 在叔丁醇钾的作用下 首先生成 $\mathrm{N}$-杂环卡宾 $\mathbf{2}, 2$ 作为亲核试剂进攻苯甲醛的 羰基碳, 生成极性反转的中间体 $\mathbf{3}$. 在中间体 $\mathbf{3}$ 中原来 的羰基碳由正电性转变成负电性, 因此中间体 3 作为亲 核试剂进攻对氟硝基苯的 $\mathrm{C}-\mathrm{F}$ 键，生成中间体 4. 然 后，中间体 4 中的氟以阴离子的形式离去，生成中间体 5. 最后， $\mathrm{N}$-杂环卡宾 2 从中间体 5 中脱除，同时脱除一 个质子, 得到产物对硝基二苯甲酮.

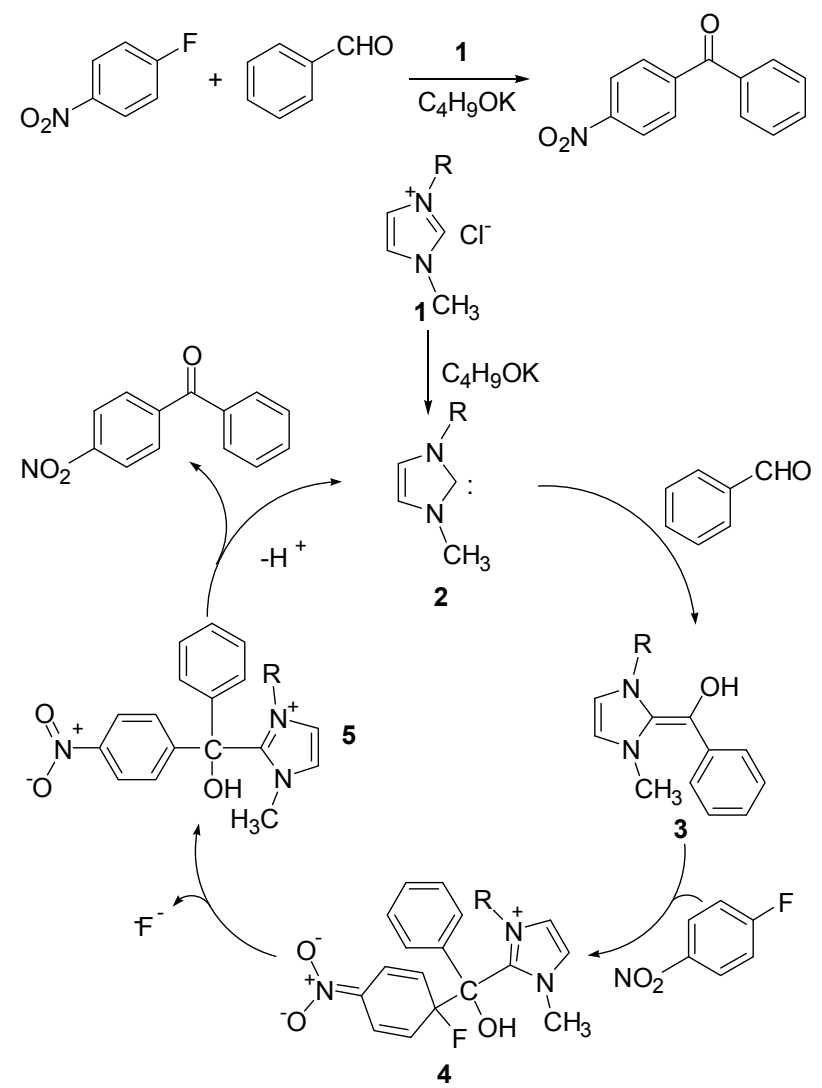

图 1 NHC 催化亲核酰基化反应的机理

Figure 1 The mechanism of nucleophilic acylation catalyzed by NHC

\section{2 离子液体对反应的影响}

将不同烷基链长的离子液体 $\mathbf{1}$ 用作 $\mathrm{NHC}$ 催化剂的 前体, 催化对氟硝基苯和苯甲醛的反应. 为了增加对氟 硝基苯的转化率, 并减少安息香缩合副反应的发生, 对 氟硝基苯和苯甲醛的物质的量比为 $1: 1.2$, 苯甲醛稍过 
量. 以 DMSO 为溶剂, 离子液体 $\mathbf{1}$ 的用量为底物对氟硝 基苯的 $33 \mathrm{~mol} \%$, 离子液体和叔丁醇钾的物质的量比为 1:4. 在 $30{ }^{\circ} \mathrm{C}$, 反应 $6 \mathrm{~h}$ 的条件下, 考察了离子液体 1 的烷基链长对催化反应的影响, 结果如表 1 所示.

表 1 离子液体对反应的影响

Table 1 The effect of the ionic liquid on the reaction

\begin{tabular}{ccc}
\hline 离子液体 $\mathbf{1}$ & 转化率 ${ }^{a} / \%$ & 收率 $/ \%$ \\
\hline $\mathrm{R}=n-\mathrm{C}_{4} \mathrm{H}_{9}$ & 85.3 & 75.3 \\
$\mathrm{R}=n-\mathrm{C}_{8} \mathrm{H}_{17}$ & 89.7 & 81.1 \\
$\mathrm{R}=n-\mathrm{C}_{12} \mathrm{H}_{25}$ & 93.0 & 88.6 \\
$\mathrm{R}=n-\mathrm{C}_{16} \mathrm{H}_{33}$ & 84.8 & 73.9 \\
$\mathrm{R}=\mathrm{CH}_{2} \mathrm{Ph}$ & 80.6 & 72.4 \\
\hline$a$ 对氟硝基苯的转化率, 下同 & &
\end{tabular}

由表 1 可知: 随着离子液体侧链长度的增加, 对氟 硝基苯的转化率和产物对硝基二苯甲酮的收率都是呈 先增加后减小的趋势. 当离子液体 $\mathbf{1}$ 的烷基链长为正十 二烷基 $\left(\mathrm{R}=n-\mathrm{C}_{12} \mathrm{H}_{25}\right)$ 时, 对氟硝基苯的转化率和产物收 率最高, 分别达到 $93.0 \%$ 和 $88.6 \%$. 这可能是因为催化 剂的催化活性受电子效应和空间效应共同作用的结果. 烷基链越长, 供电子性越大, 使生成的 $\mathrm{NHC}$ 催化剂的 亲核性越强, 催化活性增加; 但烷基链越长, 同时也增 加了空间障碍, 不利于催化反应. 因此, 选择离子液体 $1\left(\mathrm{R}=n-\mathrm{C}_{12} \mathrm{H}_{25}\right)$ 为催化剂前体, 用于下列实验的考察.

\section{3 离子液体的用量对反应的影响}

在上述反应条件下, 考察了离子液体 $1(\mathrm{R}=$ $n-\mathrm{C}_{12} \mathrm{H}_{25}$ )的用量对反应的影响, 结果如图 2 所示.

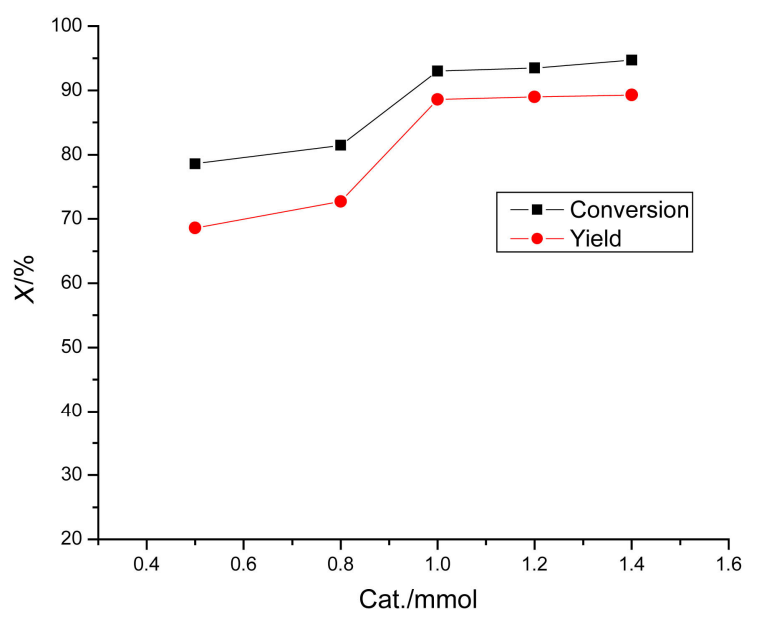

图 2 离子液体的用量对反应的影响

Figure 2 The effect of the amount of ionic liquid on the reaction

由图 2 可知, 随着离子液体用量的增加, 催化活性 中心增多，对氟硝基苯的转化率与对硝基二苯甲酮的收 率均有所提高; 当离子液体的用量为 $1 \mathrm{mmol}$, 即为底物 对氟硝基苯的 $33 \mathrm{~mol} \%$ 时, 进一步增加离子液体的用量,
转化率和收率增加的幅度不大. 因此, 最佳的离子液体 用量定为 $33 \mathrm{~mol} \%$.

\section{4 溶剂对反应的影响}

在离子液体 $1\left(\mathrm{R}=n-\mathrm{C}_{12} \mathrm{H}_{25}\right)$ 的用量为 $33 \mathrm{~mol} \%$, 离 子液体和叔丁醇钾的物质的量比为 $1: 4,30{ }^{\circ} \mathrm{C}$ 反应 $6 \mathrm{~h}$ 的条件下，考察了溶剂对反应的影响，结果如表 2 所示.

表 2 溶剂对反应的影响

Table 2 The effect of the solvent on the reaction

\begin{tabular}{cccc}
\hline 溶剂 & 极性 & 转化率 $/ \%$ & 收率 $/ \%$ \\
\hline DMSO & 7.2 & 93.0 & 88.6 \\
DMF & 6.4 & 89.9 & 68.8 \\
乙腈 & 6.2 & 85.1 & 75.4 \\
丙酮 & 5.3 & 82.6 & 64.9 \\
THF & 4.2 & 72.3 & 43.4 \\
\hline
\end{tabular}

可见，随着溶剂极性的增大，对氟硝基苯的转化率 呈递增趋势. 这可能是因为极性较大的溶剂更能很好的 溶解离子液体和碱，以及更好地稳定反应中间体，因此 催化活性高, 转化率增加. 当溶剂为 DMF 时, 虽然有较 高的转化率, 但目标产物的收率较低. 这是因为反应不 仅有安息香缩合的副产物苯偶姻的生成，而且发现有对 氟硝基苯和溶剂 DMF 反应的副产物 N,N-二甲基-4-硝基 苯胺的产生，因此降低了对硝基二苯甲酮的收率. 实验 选择极性大、转化率和收率都较高的 DMSO 为溶剂.

\section{5 碱对反应的影响}

以 $\mathrm{DMSO}$ 为溶剂，离子液体 $\mathbf{1}\left(\mathrm{R}=n-\mathrm{C}_{12} \mathrm{H}_{25}\right)$ 的用量 为 $33 \mathrm{~mol} \%$, 离子液体和碱的物质的量比为 $1: 4$, 在 $30{ }^{\circ} \mathrm{C}$ 反应 $6 \mathrm{~h}$ 的条件下，考察了碱的种类对反应的影响, 结果如表 3 所示.

表 3 碱对反应的影响

Table 3 The effect of the alkali on the reaction

\begin{tabular}{ccc}
\hline 碱 & 转化率/\% & 收率 $/ \%$ \\
\hline 叔丁醇钾 & 93.0 & 88.6 \\
$\mathrm{NaH}$ & 49.8 & 45.4 \\
$\mathrm{DBU}$ & 68.0 & 61.7 \\
三乙胺 & 16.7 & 3.0 \\
\hline
\end{tabular}

由表 3 可知, 在位阻较大的碱如叔丁醇钾或 DBU 的作用下，反应的转化率和收率相对较高，这可能是因 为生成 $\mathrm{NHC}$ 催化中心的速度较慢, 有利于反应中间体 和对氟硝基苯反应，因此对氟硝基苯的转化率相对较 高; 另外, 较强的碱对反应更有利，在小分子的弱碱如 三乙胺的作用下，因不利于 $\mathrm{NHC}$ 的生成，因此催化活 性较低, 反应的转化率和收率都低; 但另一方面, 小分 子的 $\mathrm{NaH}$ 虽为强碱，反应的转化率和收率并不高，这可 能是因为生成 $\mathrm{NHC}$ 的速度相对较快, 不利于反应中间 体和对氟硝基苯接触反应，而有利于苯甲醛的自身缩合 
反应. 总之, 大分子的强碱叔丁醇钾对反应最有利. 因 此, 反应选择叔丁醇钾为碱.

\section{6 碱的用量对反应的影响}

在上述反应条件下, 以叔丁醇钾为碱，考察了叔丁 醇钾的用量对反应的影响，结果如图 3 所示.

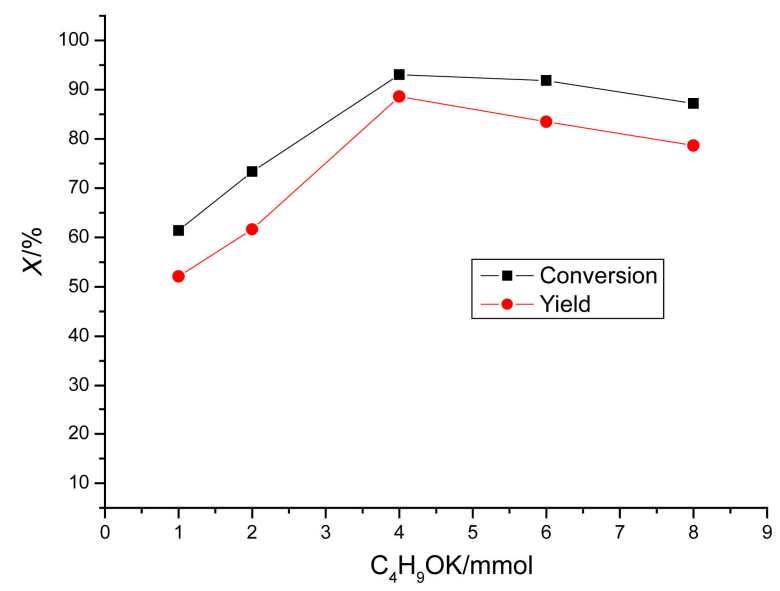

图 3 碱的用量对反应的影响

Figure 3 The effect of the amount of the alkali on the reaction

由图 3 可以看出, 当加入少量的碱时, 因生成较少 的 NHC 活性中心, 反应的转化率和收率均较低. 增加 碱的用量, 转化率和收率相应地增加, 但加入过多的碱, 转化率和收率反而开始下降. 这可能是因为过多的碱会 促进苯甲醛自身的安息香缩合副反应的发生，因此降低 了对氟硝基苯的转化率和目标产物的收率. 由图 4 可知, 当叔丁醇钾的用量为 $4 \mathrm{mmol}$, 即离子液体与碱的物质 的量比为 $1: 4$ 时, 对硝基二苯甲酮的收率最高. 因此, 最佳的碱的用量为 $4 \mathrm{mmol}$.

\section{7 温度对反应的影响}

以 DMSO 为溶剂, 离子液体 $\mathbf{1}\left(\mathrm{R}=n-\mathrm{C}_{12} \mathrm{H}_{25}\right)$ 的用量 为 $33 \mathrm{~mol} \%$, 离子液体和碱的物质的量比为 $1: 4$, 反应 时间为 $6 \mathrm{~h}$ 的条件下, 考察了温度对反应的影响, 结果 如表 4 所示.

表 4 温度对反应的影响

Table 4 The effect of the temperature on the reaction

\begin{tabular}{ccc}
\hline 温度 $/{ }^{\circ} \mathrm{C}$ & 转化率 $/ \%$ & 收率 $/ \%$ \\
\hline 30 & 93.0 & 88.6 \\
40 & 78.4 & 56.0 \\
50 & 68.4 & 49.3 \\
\hline
\end{tabular}

因为 DMSO 的熔点为 $18{ }^{\circ} \mathrm{C}$, 因此反应首先选择在 $30{ }^{\circ} \mathrm{C}$ 下进行. 由表 4 可知, 随着反应温度的升高, 对氟 硝基苯的转化率和产物对硝基二苯甲酮的收率都是降 低的. 这可能是因为温度的升高增加了苯甲酫的安息香 缩合副反应, 减少了苯甲醛和对氟硝基苯的反应; 另外,
亲核酰基化反应可能为放热反应，升高温度，对反应的 热力学不利. 因此, 确定最佳反应温度为 $30{ }^{\circ} \mathrm{C}$.

\section{8 时间对反应的影响}

在上述反应条件下, 以 $30{ }^{\circ} \mathrm{C}$ 为反应温度, 考察了 反应时间对反应的影响，结果如图 4 所示.

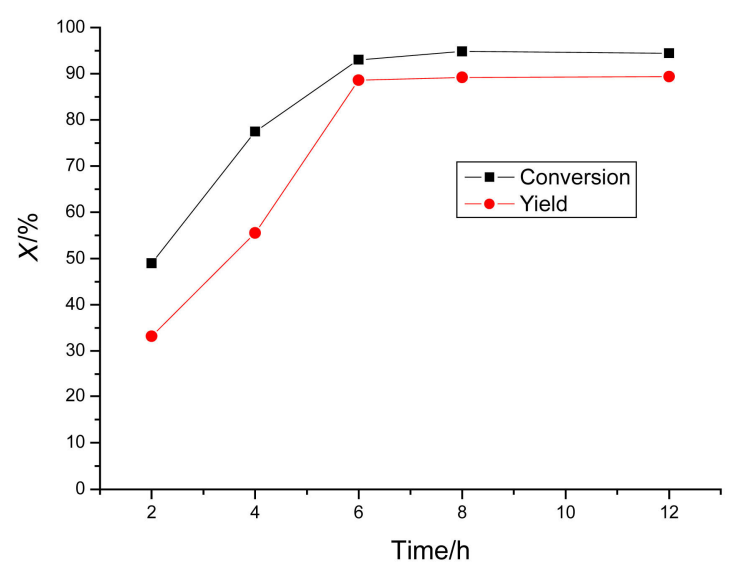

图 4 时间对反应的影响

Figure 4 The effect of the reaction time on the reaction

由图 4 可知, 随着反应时间的延长, 反应的转化率 和目标产物的收率都随之增加，当反应时间达到 $6 \mathrm{~h}$ 后， 进一步延长反应时间, 转化率与收率的增加幅度不大, 因此最佳的反应时间为 $6 \mathrm{~h}$.

\section{9 离子液体的循环利用}

反应结束后，对离子液体进行干燥处理后可直接循 环使用. 在上述选择的最佳条件下，考察了离子液体 1 $\left(\mathrm{R}=n-\mathrm{C}_{12} \mathrm{H}_{25}\right)$ 的循环使用效果, 结果如图 5 所示.

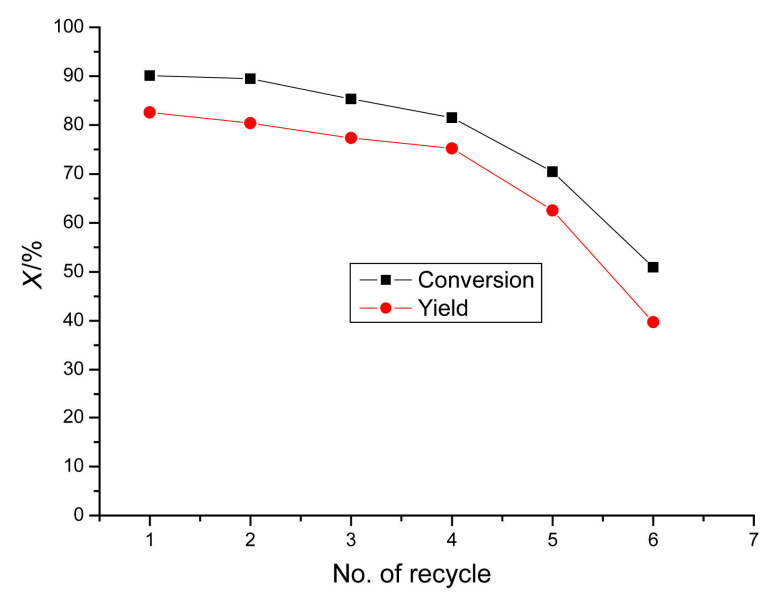

图 5 离子液体的循环使用效果

Figure 5 The recycling efficiency of the ionic liquid

由图 5 可知, 随着离子液体循环使用次数的增加, 反应的转化率和目标产物的收率有所下降, 这是因为每 次反应结束后进行的萃取操作, 产生了离子液体的流失 
所致. 离子液体重复使用 4 次, 对硝基二苯甲酮的收率 仍在 $75 \%$ 以上.

\subsection{0 底物对反应的影响}

在上述选择的最佳条件下, 考察了不同芳香氟化物 和系列醛的亲核酰基化反应，结果如表 5 所示.

由表 5 可以看出, 含吸电子基团的芳香氟化物对氟 硝基苯和对氟苯甲腈都可以很好地和醛发生反应, 生成 相应的芳香酮(Entries 1 5); 而含供电子基团的芳香氟 化物对氟甲苯和苯甲醛的反应, 没有得到相应的芳香酮 (Entry 6). 这是因为该类型的反应是一个亲核机理的反 应, 吸电子基团的存在降低了芳环的电子云密度, 有利 于亲核试剂的进攻. 因此, 该类型的反应适用于将酰基 直接引入到芳环的缺电子位置, 制备出含吸电子基团的 芳香酮类化合物. 对于该类型反应中底物醛的适用性, 不仅适用于芳香醛, 而且适用于脂肪醛(Entry 4); 但是 脂肪醛的酰基化产物收率明显较低, 这是因为在碱性条 件下丁醛的自身着醛缩合反应较强烈的原因. 另外, 由 Entries 1 3 和 Entry 7 可知, 含供电子基的芳香醛对反 应有利，这可能是因为供电子基的存在增加了反应中间 体 3 的亲核性，从而有利于亲核酰基化反应.

表 5 底物对反应的影响

Table 5 The effect of the substrate on the reaction

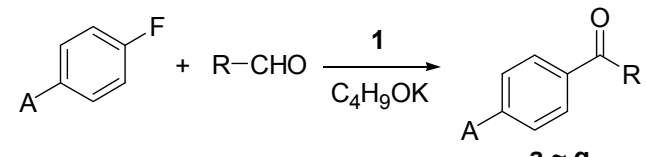

$\mathbf{a} \sim \mathbf{g}$

\begin{tabular}{cclccc}
\hline Entry & $\mathrm{A}$ & \multicolumn{1}{c}{$\mathrm{R}$} & 产物 & 转化率 ${ }^{b} / \%$ & 收率/\% \\
\hline 1 & $\mathrm{NO}_{2}$ & $\mathrm{C}_{6} \mathrm{H}_{5}$ & $\mathbf{a}$ & 93.0 & 88.6 \\
2 & $\mathrm{NO}_{2}$ & $4-\mathrm{MeOC}_{6} \mathrm{H}_{4}$ & $\mathbf{b}$ & 96.3 & 76.5 \\
3 & $\mathrm{NO}_{2}$ & $4-\mathrm{MeC}_{6} \mathrm{H}_{4}$ & $\mathbf{c}$ & 95.2 & 73.2 \\
4 & $\mathrm{NO}_{2}$ & $n-\mathrm{C}_{3} \mathrm{H}_{7}$ & $\mathbf{d}$ & 61.5 & 43.4 \\
5 & $\mathrm{CN}$ & $\mathrm{C}_{6} \mathrm{H}_{5}$ & $\mathbf{e}$ & 88.7 & 62.5 \\
6 & $\mathrm{CH}_{3}$ & $\mathrm{C}_{6} \mathrm{H}_{5}$ & $\mathbf{f}$ & - & - \\
7 & $\mathrm{NO}_{2}$ & $4-\mathrm{NO}_{2} \mathrm{C}_{6} \mathrm{H}_{4}$ & $\mathbf{g}$ & - & - \\
\hline
\end{tabular}

${ }^{b}$ 芳香氟化物的转化率.

\section{2 结论}

以咪唑类离子液体 $\mathbf{1}$ 为 $\mathrm{NHC}$ 催化剂的前体, 应用 于催化含吸电子基团的芳香氟化物和醛之间的亲核酰 基化反应，成功地制备出含有吸电子基团的芳香酮类化 合物, 并实现了催化剂的循环使用. 对氟硝基苯和苯甲 醛的反应制备对硝基二苯甲酮，优化的反应条件为：以 DMSO 为溶剂、离子液体 $\mathbf{1}\left(\mathrm{R}=n-\mathrm{C}_{12} \mathrm{H}_{25}\right)$ 为催化剂前体、 叔丁醇钾为碱, 离子液体的用量为对氟硝基苯的 33 $\mathrm{mol} \%$, 离子液体和叔丁醇钾的摩尔比为 $1: 4$, 反应温 度为 $30{ }^{\circ} \mathrm{C}$, 反应时间为 $6 \mathrm{~h}$. 在上述优化的反应条件下,
对氟硝基苯的转化率为 $93.0 \%$, 对硝基二苯甲酮的收率 为 $88.6 \%$. 离子液体循环使用 4 次，对硝基二苯甲酮的 收率仍在 $75 \%$ 以上. 本研究为直接将酰基引入到缺电子 的芳环上、制备含有吸电子基团的芳香酮提供了一条环 境友好的新途径.

\section{3 实验部分}

\section{1 仪器与试剂}

德国 Brucker 公司 AV500 核磁共振波谱仪, TMS 为 内标, $\mathrm{CDCl}_{3}$ 为溶剂; 上海精密科学仪器有限公司 $\mathrm{X}-4$ 显微熔点仪; 日本岛津 LC-20A 高效液相色谱仪.

青岛海洋化工有限公司 GF254 薄层层析硅胶, 200 300 目柱层层析硅胶; 其余所用试剂均为分析纯 试剂.

\section{2 实验方法}

\subsection{1 离子液体的制备}

向装有回流冷凝管的 $100 \mathrm{~mL}$ 单口烧瓶中加入 0.05 $\mathrm{mol} \mathrm{N}$-甲基咪唑、0.06 mol 氯代正丁烷和 $10 \mathrm{~mL}$ 甲醇. 升 温至 $70 \sim 80{ }^{\circ} \mathrm{C}$, 回流反应 $24 \mathrm{~h}$. 减压蒸除溶剂, 未反应 的氯代正丁烷用石蜡吸收. 用乙酸乙酯洗涤剩余物 2 次, 旋蒸除去乙酸乙酯. 干燥，得到黄色粘稠离子液体 1 $\left(\mathrm{R}=n-\mathrm{C}_{4} \mathrm{H}_{9}\right)$.

把氯代正丁烷分别换成氯代正辛烷、氯代正十二 烷、氯代正十八烷和苄基氯，通过上述相同的实验操作， 制备出其他含不同侧链的的咪唑类离子液体 $1(\mathrm{R}=$ $n-\mathrm{C}_{8} \mathrm{H}_{17}, n-\mathrm{C}_{12} \mathrm{H}_{25}, n-\mathrm{C}_{16} \mathrm{H}_{33}, \mathrm{CH}_{2} \mathrm{Ph}$ ).

\section{2 .2 亲核酰基化反应}

向装有回流冷凝管的 $100 \mathrm{~mL}$ 三口烧瓶中依次加入 $3 \mathrm{mmol}$ 芳基氟化物、3.6 $\mathrm{mmol}$ 芳香醛或脂肪醛、一定 量的咪唑类离子液体 $\mathbf{1}$ 和 $20 \mathrm{~mL}$ 溶剂. 搅拌 $5 \mathrm{~min}$ 后，向 三口烧瓶中再加入一定量的碱. 在一定温度下摚拌反应 一定时间，可通过 TLC 跟踪反应.

反应结束后，向反应液中加入冰水. 用乙酸乙酯萃 取有机物 2 次，合并萃取液. 经水洗、盐洗、干燥后，减 压蒸除溶剂. 利用柱层析分离出粗产品, 用乙醇重结晶, 得到无色针状晶体产物. 溶于水的离子液体经真空干燥 后可循环使用.

对硝基二苯甲酮(a): m.p. $136 \sim 138{ }^{\circ} \mathrm{C}$; ${ }^{1} \mathrm{H}$ NMR $\left(\mathrm{CDCl}_{3}, 500 \mathrm{MHz}\right) \delta: 7.53(\mathrm{t}, J=8.0 \mathrm{~Hz}, 2 \mathrm{H}), 7.66(\mathrm{t}, J=$ $8.0 \mathrm{~Hz}, 1 \mathrm{H}), 7.81(\mathrm{~d}, J=8.0 \mathrm{~Hz}, 2 \mathrm{H}), 7.94(\mathrm{~d}, J=8.5 \mathrm{~Hz}$, $2 \mathrm{H}), 8.35(\mathrm{~d}, J=8.5 \mathrm{~Hz}, 2 \mathrm{H}) ;{ }^{13} \mathrm{C} \mathrm{NMR}\left(\mathrm{CDCl}_{3}, 125 \mathrm{MHz}\right)$ $\delta: 123.54,128.68,130.09,130.68,133.46,136.30,142.90$, $149.85,194.78$.

4-甲氧基-4'-硝基二苯甲酮(b)：m.p. $124 \sim 126{ }^{\circ} \mathrm{C}$; 
${ }^{1} \mathrm{H}$ NMR $\left(\mathrm{CDCl}_{3}, 500 \mathrm{MHz}\right) \delta: 8.34(\mathrm{~d}, J=8.5 \mathrm{~Hz}, 2 \mathrm{H})$, $7.88(\mathrm{~d}, J=8.5 \mathrm{~Hz}, 2 \mathrm{H}), 7.81(\mathrm{~d}, J=8.5 \mathrm{~Hz}, 2 \mathrm{H}), 6.96(\mathrm{~d}$, $J=9.0 \mathrm{~Hz}, 2 \mathrm{H}), 3.91(\mathrm{~s}, 3 \mathrm{H}) ;{ }^{13} \mathrm{C} \mathrm{NMR}\left(\mathrm{CDCl}_{3}, 125 \mathrm{MHz}\right)$ $\delta:$ 55.61, 113.98, 123.48, 128.94, 130.32, 132.65, 143.80, $149.53,164.02,193.46$.

4-甲基-4'-硝基二苯甲酮(c): m.p. $122 \sim 125{ }^{\circ} \mathrm{C} ;{ }^{1} \mathrm{H}$ NMR $\left(\mathrm{CDCl}_{3}, 500 \mathrm{MHz}\right) \delta: 8.34(\mathrm{~d}, J=8.5 \mathrm{~Hz}, 2 \mathrm{H}), 7.91$ (d, $J=9.0 \mathrm{~Hz}, 2 \mathrm{H}), 7.71(\mathrm{~d}, J=8.5 \mathrm{~Hz}, 2 \mathrm{H}), 7.32$ (d, $J=$ $8.0 \mathrm{~Hz}, 2 \mathrm{H}), 2.46(\mathrm{~s}, 3 \mathrm{H}) ;{ }^{13} \mathrm{C} \mathrm{NMR}\left(\mathrm{CDCl}_{3}, 125 \mathrm{MHz}\right) \delta$ : $21.75,123.49,129.38,130.32,130.54,133.66,143.34$, 144.57, 149.70, 194.50.

对氧基二苯甲酮(d): m.p. $111 \sim 113{ }^{\circ} \mathrm{C} ;{ }^{1} \mathrm{H}$ NMR $\left(\mathrm{CDCl}_{3}, 500 \mathrm{MHz}\right) \delta$ : $7.88(\mathrm{~d}, J=8.0 \mathrm{~Hz}, 2 \mathrm{H}), 7.78 \sim 7.80$ (m, 4H), $7.64(\mathrm{t}, J=7.5 \mathrm{~Hz}, 1 \mathrm{H}), 7.52(\mathrm{t}, J=7.5 \mathrm{~Hz}, 2 \mathrm{H})$; ${ }^{13} \mathrm{C}$ NMR $\left(\mathrm{CDCl}_{3}, 125 \mathrm{MHz}\right) \delta: 115.67,117.99,128.63$, 130.05, 130.22, 132.16, 133.31, 136.34, 141.24, 195.02.

对硝基苯丁酮(e): m.p. $64 \sim 65{ }^{\circ} \mathrm{C} ;{ }^{1} \mathrm{H}$ NMR $\left(\mathrm{CDCl}_{3}\right.$, $500 \mathrm{MHz}) \delta: 8.32(\mathrm{~d}, J=9.0 \mathrm{~Hz}, 2 \mathrm{H}), 8.12(\mathrm{~d}, J=8.5 \mathrm{~Hz}$, $2 \mathrm{H}), 3.02(\mathrm{t}, J=7.5 \mathrm{~Hz}, 2 \mathrm{H}), 1.77 \sim 1.84(\mathrm{~m}, 2 \mathrm{H}), 1.04(\mathrm{t}$, $J=7.5 \mathrm{~Hz}, 3 \mathrm{H}) ;{ }^{13} \mathrm{C} \mathrm{NMR}\left(\mathrm{CDCl}_{3}, 125 \mathrm{MHz}\right) \delta: 13.66$, 17.39, 40.97, 123.79, 128.98, 141.46, 150.19, 198.64.

辅助材料(Supporting Information) 产物 $\mathbf{a} \sim \mathbf{e}$ 的核磁 谱图. 这些材料可以免费从本刊网站(http://sioc-journal. $\mathrm{cn} /$ )上下载.

\section{References}

[1] Wöhler, F.; Liebig, J. Ann. Pharm. 1832, 3, 249.

[2] Lapworth, A. J. J. Chem. Soc. 1903, 83, 995.

[3] Ukai, T.; Tanaka, R.; Dokawa, T. J. Pharm. Soc. Jpn. 1943, 63, 296.

[4] Arduengo, A. J.; Harlow, R. L.; Kline, M. J. Am. Chem. Soc. 1991, $113,361$.

[5] Yu, H.-Z.; Fu, Y.; Liu, L.; Guo, Q.-X. Chin. J. Org. Chem. 2007,
27, 545 (in Chinese).

(于海珠, 傅尧, 刘否, 郭庆祥, 有机化学, 2007, 27, 545.)

[6] Sun, X.-Y.; Wu, J. Chin. J. Org. Chem. 2006, 26, 745 (in Chinese). (孙小宇, 吴款, 有机化学, 2006, 26, 745.)

[7] Qu, M.-N.; He, J.-M. Chin. J. Org. Chem. 2011, 31, 1388 (in Chinese).

(屈孟男, 何金梅, 有机化学, 2011, 31, 1388.)

[8] Ca, X.-H.; Xie, B. Chin. J. Appl. Chem. 2013, 30, 123 (in Chinese). (蔡下华, 谢兵, 应用化学, 2013, 30, 123.)

[9] Bugant, X.; Glorius, F. Chem. Soc. Rev. 2012, 41, 3511.

[10] Chai, H. X.; Li, J. R.; Yang, L. P.; Liu, M. X.; Yang, D. L.; Zhang, Q.; Shi, D. X. Chin. J. Chem. 2014, 32, 865.

[11] Enders, D.; Kallfass, U. Angew. Chem., Int. Ed. 2002, 41, 1743.

[12] Mennen, S. M.; Gipson, J. D.; Kim, Y. R.; Miller, S. J. J. Am. Chem. Soc. 2005, 127, 1654.

[13] Enders, D.; Niemeier, O.; Balensiefer, T. Angew. Chem., Int. Ed. 2006, 45, 1463.

[14] Liu, Q.; Rovis, T. J. Am. Chem. Soc. 2006, 128, 2552.

[15] Li, Y.; Shi, F. Q.; He Q. L.; You, S. L. Org. Lett. 2009, 11, 3182.

[16] Xie, Y.-M.; Wu, J.; Chao, X.-J. Chem. Bioeng. 2011, 28, 10 (in Chinese).

(谢益明, 吴杰, 晁雪静, 化学与生物工程, 2011, 28, 10.)

[17] He, M., Gerson, J. U.; Jeffrey, W. B. J. Am. Chem. Soc. 2006, 128, 15088.

[18] Yu, F. L.; Jiang, J. J.; Zhao, D. M.; Xie, C. X.; Yu, S. T. RSC Adv. 2013, 3, 3996.

[19] Yu, F.-L.; Dou, Y.-T.; Zhao, D.-M.; Xie, C.-X.; Yu, S.-T. Chin. J. Org. Chem. 2014, 34, 1190 (in Chinese).

(于凤丽, 窦有涛, 赵冬梅, 解从霞, 于世涛, 有机化学, 2014, 34, 1190.)

[20] Yu, F.-L.; Zhao, D.-M.; Xie, C.-X.; Yu, S.-T. Chemistry 2014, 76, 712 (in Chinese) (于凤丽, 赵冬梅, 解从霞, 于世涛, 化学通报, 2014, 76, 712.)

[21] Grasa, G. A.; Kissling, R. M.; Nolan, S. P. Org. Lett. 2002, 4, 3583.

[22] Suzuki, Y.; Yamauchi, K.; Muramatsu, K.; Sato, M. Chem. Commun. 2004, 2770.

[23] Kano, T.; Sasaki, K.; Maruoka, K. Org. Lett. 2005, 7, 1347.

[24] Suzuki, Y.; Toyota, T.; Imada, F.; Satoa, M.; Miyashita, A. Chem. Commun. 2003, 1314.

[25] Suzuki, Y.; Ota, S.; Fukuta, Y.; Ueda, Y.; Sato, M. J. Org. Chem. 2008, 73, 2420.

[26] Suzuki, Y.; Toyota, T.; Miyashita, A.; Sato, M. Chem. Pharm. Bull. 2006, 54, 1653. 